\title{
Exhaust gas temperature measurements in diagnostic examination of naval gas turbine engines
}

\author{
Part III \\ Diagnostic and operating tolerances
}

\author{
Zbigniew Korczewski, Prof. \\ Gdansk University of Technology
}

\section{ABSTRACT}

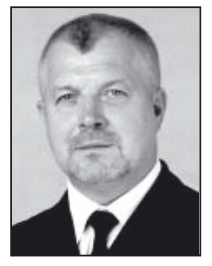

The third part of the article presents a method for detecting failures of the automatic engine control system with the aid of an exhaust gas temperature setter, specially designed and machined for this purpose. It also presents a procedure of identifying the operating tolerances and determining the diagnostic tolerances for the exhaust gas temperature recorded in the naval turbine engine during the start-up and acceleration processes. The diagnostic tolerances were determined using the statistic inference, based on the hypothesis about the normal distribution of the starting exhaust gas temperature dispersion at the initial time of engine operation. The above hypothesis was verified using the non-parametric statistic test $\chi^{2}$ for examining the consistency of the empirical distribution with the assumed normal distribution. As a result of the examination, satisfactory convergence of the compared distributions was obtained which made the basis for assuming the three-sigma limits of the diagnostic tolerance for the analysed engine control parameter.

Key words: technical diagnostics; naval turbine engines; exhaust gas temperature; operating and diagnostic tolerances

\section{INTRODUCTION}

The general assessment of the technical state of a naval turbine engine can be done based on the values of basic parameters, such as power, specific fuel consumption, rotational speeds of rotating units, and/or mass flow rates of the delivered fuel or the thermodynamic medium flowing through the engine. All these parameters characterise, in a general way, the quality of engine operation $[1,2,3,6]$. However, in case of most serial engines, the above parameters cannot be directly measured (their measurement is extremely complicated and/or economically unjustified) in conditions of engine operation in the naval power plant. Moreover, these parameters do not deliver sufficiently precise information on the real course of the physical processes taking place in basic functional modules of the engine (flow part, kinematic system, fuel installation, automatic control system, etc.) $[4,5,7,8,14]$. That is why the operational diagnostics of turbine engines makes use of a set of auxiliary control parameters which indirectly define the basic parameters and, additionally, provide opportunities for indicating the areas in which the largest energy losses are observed, as well as for identifying and localising known and recognisable failures of engine sub-assemblies and constructional elements during engine operation.
The real value of each control parameter differs from its calculated value, which results from differences in machining (inaccuracies of the realised technological process), the action of external agents (temperature, humidity, dust, vibrations, sea undulation, etc.), impurities, and/or wear and tear of constructional elements (reversible and irreversible changes of the surface layer) $[7,8,9]$. These actions are of random nature (random events) and, as a consequence, generate random changes of values of the structure parameters $[2,7]$. Their effect on the technical state of the engine can also be observed as changes of the probability distribution of the occurrence of these parameters - the expected value and the average deviation (dispersion around the mean value for the set of engines in operation). The smaller the changes of the expected value and the mean deviation of the control parameter, the higher the correctness of engine operation and, consequently, its lifetime and reliability.

The correctness of operation of a constructional module or unit, as well as that of the entire turbine engine, is defined by the tolerances of parameters which reflect the most desirable and permissible courses of the realised physical processes:

- Operating tolerances (maximal permissible) - determined by the producer (designer) based on the tests done on prototype copies. They define precisely the maximum ranges within 
which the values of the control parameters are to be kept during the engine operation process to secure that the engine performance parameters do not drop below permissible levels, the engine operation is undisturbed and reliable, and that the technical durability is good and in line with the limits of the operating potential for particular hours and days. When the value of any control parameter exceeds the set operating tolerance limits, it is a signal of unacceptable disturbance of energy conversion processes realised in the engine, which threats with its failure (unstable operation of the compressor, for instance) $[3,7,8,11,12,14]$.

- Diagnostic tolerances - determined via statistical inference of the results of the tests done on a sufficiently numerous set of new and correctly adjusted engines (revealing full technical ability), compared with the results of operating tests of the same engines is successive stages of their use, provided that they still maintain a good functioning level, which is assessed from changes of basic parameters. During the time of engine operation the values of the control parameters shift towards the diagnostic tolerance limits, which signals the symptoms of small changes of engine's technical state, characteristic for the "approaching" state of inability. At the same time, the above changes of the basic parameters reflect the dependence of the technical state of the engine on the time of its operation, which manifests itself as changes of the expected values of the control parameters and their average deviations.

Consequently, a conclusion can be formulated that the tolerances of the control parameters for diagnostic purposes should be much smaller (more restrictive) that the operating tolerances.

\section{IDENTIFYING FAILURES OF THE AUTOMATICS SYSTEM (OPERATING TOLERANCES)}

Some most typical operating failures of the engine automatics system can be identified using simulation analyses done with the aid of specially designed setters (testers) on the non-operating engine [15]. These devices control the correctness of operation of the engine protection system against the excessive increase of the exhaust gas temperature. The tests aim at checking correct threshold settings for the (maximal) exhaust gas temperatures behind the exhaust gas generator, and the temperature grow rates at which the final control units in the automatics system open:

- the fuel overflow valve (ZPP),

- the main fuel valve (ZGP),

- the immediate (emergency) engine switch-off valve (ZA).

The values determined in the above way are considered the limits of the operating tolerance zone for permissible changes of parameters in the process of engine start-up and acceleration. Sample results of these tests are shown in Fig. 1 in the form of simulated dynamic time-histories of exhaust gas temperature changes. To assess the ability of the examined engine, the timehistories obtained from the simulation test are compared with the real curves recorded during earlier start-ups (see: part II - Unsteady states).

The comparison analysis of the results recorded during many years of operation of the UGT type ZORYA naval engines which were the object of diagnostic supervision [15] has made it possible to recognise characteristic thresholds for initiating certain actions of the protection system. These thresholds are

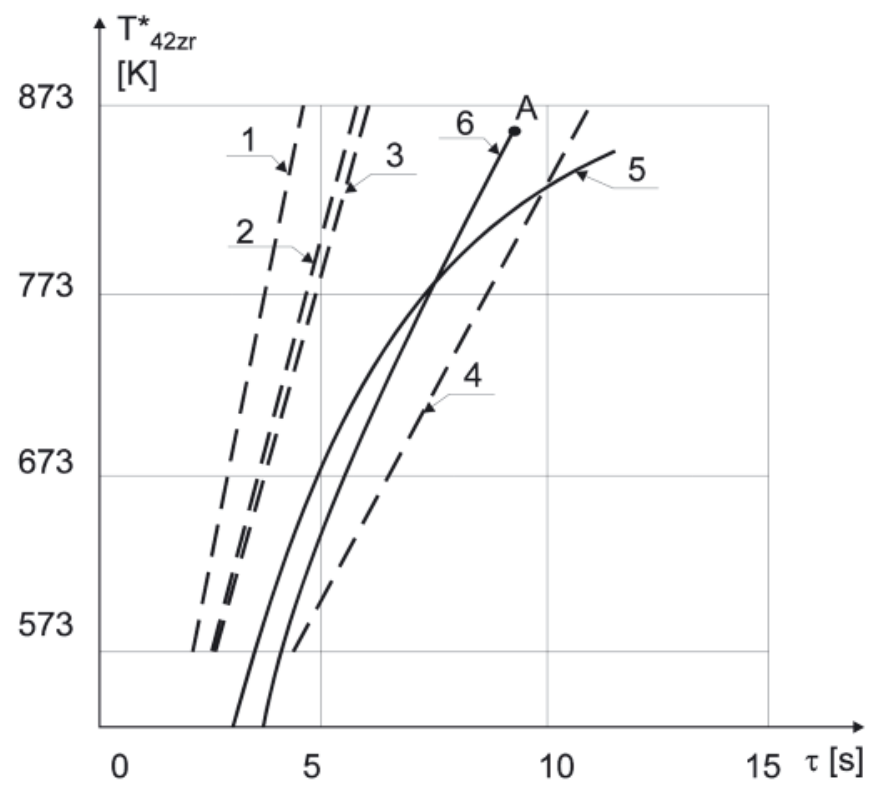

Fig. 1. Simulated (dashed lines) and real (solid lines) time-histories of the exhaust gas temperature recorded behind the low pressure turbine during diagnostic start-up tests of the UGT type ZORYA engine.

1 - input function $d T_{42}^{*} / d \tau=112 \mathrm{~K} / \mathrm{s}-Z P P(-), Z G P(+), Z A(+)$;

2 - input function $d T_{42}^{*} / d \tau=88 \mathrm{~K} / \mathrm{s}-Z P P(-), Z G P(+), Z A(+)$;

3 -input function $d T_{42}^{*} / d \tau=84 \mathrm{~K} / \mathrm{s}-Z P P(+), Z G P(+), Z A(+)$

4 - input function $d T_{42}^{*} / d \tau=48 \mathrm{~K} / \mathrm{s}-\mathrm{ZPP}(-), Z G P(-), Z A(-)$;

5 - successful engine start-up $d T_{42}^{*} / d \tau=64 \mathrm{~K} / \mathrm{s}$;

6-unsuccessful start-up $d T_{42}^{*} / d \tau=82 \mathrm{~K} / \mathrm{s}$;

$\boldsymbol{A}$ - automatic engine switch-off

considered the limits of the operating tolerance zone for the time derivative of the averaged exhaust gas temperature behind the exhaust gas generator:

- $\mathrm{dT}_{42}^{*} / \mathrm{d} \tau>80 \mathrm{~K} / \mathrm{s}$ - the engine is automatically switched-off - ZPP(+), ZGP(+), ZA(+);

- $\mathrm{dT}_{42}^{*} / \mathrm{d} \tau=50 \div 80 \mathrm{~K} / \mathrm{s}$ - the engine can be started with automatic fuel dosage - ZPP(+), ZGP(-), ZA(-);

- $\mathrm{dT}_{42}^{*} / \mathrm{d} \tau<50 \mathrm{~K} / \mathrm{s}-$ the engine is starter automatically without the action of the protection system - ZPP(-), ZGP(-), ZA(-).

Having analysed the data shown in Fig. 1 we can conclude that the system of thermal engine protection is out of order in this case. The fuel overflow valve (ZPP) does not work, irrespective of the rate of exhaust gas temperature grow (curves 1 and 2). After a detailed analysis of the recorded symptoms of engine inability, the damaged engine automatics elements were localised and replaced. The next simulation tests confirmed the correctness of the formulated diagnosis, which is illustrated in Fig. 1 by the line 3 recorded after removing the defect.

It also results from the performed tests that the system has the zone of insensitivity ranging between 273 and $593 \mathrm{~K}$, irrespective of the dynamics of exhaust gas temperature grow.

\section{METHOD OF DEFINING DIAGNOSTIC TOLERANCES}

Measurements of the control parameters which characterise the quality of the turbine engine operation in the processes of start-up, acceleration and deceleration of rotor units, the operation at steady-load ranges, and the engine switch-off are done in the very initial stage of engine use, directly after its installation in the power plant room $[14,15]$. Discrete values 
of the diagnostic parameters recorded in these measurements are modelled as continuous and one-dimensional random variables with the certain probability distribution, expected value, and variance being the measure of the dispersion of the measured results. In this situation the diagnostic tolerance calculation method is mainly limited to finding the form of the distribution of the dispersion of the observed discrete values of the diagnostic parameter around the average value for the entire population of engines in operation, and then to determining the parameters of this distribution. One of methods making use of the statistic inference to find the form of the distribution of the random variable consists in verifying the hypothesis that the examined distribution of the discrete values of the diagnostic parameter can be approximated using an assumed theoretical distribution which is described by a known random variable probability density function at time $t=0[2,10]$. Once the form of the function of the theoretical distribution and its characteristic parameters (the expected value $\mathrm{m}$ and the average deviation $\sigma$ ) are known, we can determine the interval of tolerance for the values of the diagnostic parameters, for instance $m \pm 3 \sigma$ for the normal distribution.

A method for determining the limits of the diagnostic tolerance zone for the rate of grow of the exhaust gas temperature behind the exhaust gas generator in the start-up process ${ }^{1)}, \mathrm{dT}_{42}^{*} / \mathrm{d} \tau$, which take into account the unrepeatability of production and the effect of external operating agents, will be demonstrated on the population of 46 UGT3000 type Zorya turbine engines which were introduced to operation in naval power plants $[14,15]$. Table 1 collects a set of realisations of the numerical values of the starting temperatures recorded in the start-up process of the new engines (or directly after general overhaul) in the state of full technical ability (the values are reduced to normal atmospheric conditions) $[3,13]$.
$\mathbf{T a b}_{*}$ 1. Set of realisations of numerical values of the starting temperature $d T_{42}^{*} / d \tau$ (random variable $\left.X(t)\right)$ recorded during the start-up in the initial time of operation of UGT3000 engines installed in naval power plants

\begin{tabular}{|c|c|c|c|c|c|}
\hline Item & $\mathrm{x}_{\mathrm{i}}[\mathrm{K} / \mathrm{s}]$ & $\mathrm{n}_{\mathrm{i}}$ & Item & $\mathrm{x}_{\mathrm{i}}[\mathrm{K} / \mathrm{s}]$ & $\mathrm{n}_{\mathrm{i}}$ \\
\hline 1 & 12 & 1 & 8 & 20 & 5 \\
\hline 2 & 13 & 2 & 9 & 21 & 4 \\
\hline 3 & 14 & 3 & 10 & 22 & 3 \\
\hline 4 & 15 & 4 & 11 & 23 & 3 \\
\hline 5 & 16 & 4 & 12 & 24 & 3 \\
\hline 6 & 17 & 5 & 13 & 25 & 2 \\
\hline 7 & 19 & 6 & 14 & 29 & 1 \\
\hline
\end{tabular}

Assuming that the distribution of the values of the analysed diagnostic parameter in time $\mathrm{t}=0$ is normal, which is confirmed by diagnostic tests of aircraft engines [2], a zero hypothesis $\mathrm{H}_{0}$ is formulated that the distribution function of the empirical distribution shown in Table 1 is consistent with the normal distribution function. The zero hypothesis was verified using the non-parametric statistical test $\chi^{2}$, which does not require the information on normal distribution parameters, i.e. the expected value $m$ and the variance $\sigma^{2}[10]$. In this situation the statistical estimation of the expected value and the variance for the calculated statistics is made based on the arithmetic mean $\bar{x}$ and the standard deviation $\bar{\sigma}$ of the measured results.

For testing purposes, the interval within which the values of the random variable are included $\mathrm{X}(\mathrm{t})=\left\{\mathrm{x}_{\mathrm{i}}\right\}$ was divided into six equal sub-intervals of $3,0 \mathrm{~K} / \mathrm{s}$ in length (the widths of the intervals do not have to be equal) - Table 2 . The limits of the intervals were chosen in such a way that the numbers of the results of measurement in successive intervals were not excessively small (5-8 results in each interval as the minimum).

Tab. 2. Scheme of $\chi_{o}^{2}$ statistics calculations

\begin{tabular}{|c|c|c|c|c|c|}
\hline $\begin{array}{l}\text { Number of } \\
\text { interval }\end{array}$ & $\begin{array}{c}\text { Limits of } \\
\text { intervals } \\
\mathbf{x}_{\mathbf{i}}[\mathbf{K} / \mathbf{s}]\end{array}$ & \multicolumn{2}{|c|}{$\begin{array}{c}\text { Number of results } \\
\text { of measurement } \\
\text { in i-th interval } \\
n_{i}\end{array}$} & $\begin{array}{l}\text { Normalised } \\
\text { limits of } \\
\text { intervals } \\
\mathbf{z}_{\mathbf{i}}=\frac{\mathbf{x}_{\mathbf{i}}-\overline{\mathbf{x}}}{\sigma}\end{array}$ & $\begin{array}{l}\text { Value of Laplace function } \\
\text { for beginnings of } \\
\text { intervals } \\
\Phi p(z i)\end{array}$ \\
\hline 1 & 2 & \multicolumn{2}{|l|}{3} & 4 & 5 \\
\hline 1 & $11 \ldots 14$ & \multicolumn{2}{|l|}{6} & $-\infty \ldots-1.32$ & -0.5 \\
\hline 2 & $14 \ldots 17$ & \multicolumn{2}{|c|}{13} & $-1.32 \ldots-0.52$ & -0.4064 \\
\hline 3 & $17 \ldots 20$ & \multicolumn{2}{|c|}{11} & $-0.52 \ldots+0.27$ & -0.1985 \\
\hline 4 & $20 \ldots 23$ & \multicolumn{2}{|c|}{10} & $0.27 \ldots 1.06$ & 0.1065 \\
\hline 5 & $\boldsymbol{\Gamma}^{23 \ldots 26}$ & \multicolumn{2}{|c|}{5} & $1.06 \ldots 1.86$ & 0.3507 \\
\hline 6 & 乙26...29 & \multicolumn{2}{|c|}{1} & $1.86 \ldots+\infty$ & 0.4687 \\
\hline Sum & - & \multicolumn{2}{|l|}{46} & - & - \\
\hline $\begin{array}{l}\text { Number of } \\
\text { interval }\end{array}$ & \multicolumn{2}{|c|}{$\begin{array}{c}\text { Theoretical probability } \\
\text { to obtain the result } \\
\text { from i-th interval } \\
p_{i}=\Phi_{p}\left(z_{i+1}\right)-\Phi_{p}\left(z_{i}\right)\end{array}$} & \multicolumn{2}{|c|}{$\begin{array}{l}\text { Theoretical number of } \\
\text { results in i-th interval } \\
\qquad n \cdot p_{i}\end{array}$} & $\begin{array}{l}\text { Value of statistics for i-th } \\
\text { interval } \\
\chi_{i}^{2}=\frac{\left(\mathbf{n}_{i}-\mathbf{n} \cdot \mathbf{p}_{\mathrm{i}}\right)^{2}}{\mathbf{n} \cdot \mathbf{p}_{\mathrm{i}}}\end{array}$ \\
\hline 1 & \multicolumn{2}{|c|}{6} & & 7 & 8 \\
\hline 1 & \multicolumn{2}{|c|}{0.0936} & & 4.3056 & 0.6668 \\
\hline 2 & \multicolumn{2}{|c|}{0.2079} & & 9.5634 & 1.2349 \\
\hline 3 & \multicolumn{2}{|c|}{0.3050} & & 14.0300 & 0.6544 \\
\hline 4 & \multirow{2}{*}{\multicolumn{2}{|c|}{0.2442}} & & 11.2332 & 0.1354 \\
\hline 5 & \multirow{2}{*}{\multicolumn{2}{|c|}{$\frac{-}{0.1493}$}} & & - & - \\
\hline 6 & & & & 6.8678 & 0.1097 \\
\hline Sum & \multicolumn{2}{|c|}{1.0000} & & 46 & 2.8012 \\
\hline
\end{tabular}

1) Further in the text, more briefly referred to as the "starting temperature". 
If the number of the results of measurement in the interval is smaller than 5, this interval is to be linked with one of the neighbouring intervals [10].

In successive steps we calculate:

- the normalised limits of the intervals with respect to $\bar{x}$, recalculated to $\bar{\sigma}$ units:

$$
\mathrm{z}_{\mathrm{i}}=\frac{\mathrm{x}_{\mathrm{i}}-\overline{\mathrm{x}}}{\bar{\sigma}}
$$

The beginning of the first interval is $-\infty$, while the end of the last interval is $+\infty$. The end of the previous interval is simultaneously the beginning of the next interval;

- the normalised Laplace function of the normal distribution for the beginnings $\Phi p(z i)$ of the intervals - from statistical tables [10];

- the theoretical probability to obtain the result from the i-th interval:

$$
\mathrm{p}_{\mathrm{i}}=\Phi_{\mathrm{p}}\left(\mathrm{z}_{\mathrm{i}+1}\right)-\Phi_{\mathrm{p}}\left(\mathrm{z}_{\mathrm{i}}\right)
$$

- the theoretical number of results in the i-th interval: $n \cdot p_{i}$;

- the $\chi_{\mathrm{i}}^{2}$ statistics for each interval:

$$
\chi_{\mathrm{i}}^{2}=\frac{\left(\mathrm{n}_{\mathrm{i}}-\mathrm{n} \cdot \mathrm{p}_{\mathrm{i}}\right)^{2}}{\mathrm{n} \cdot \mathrm{p}_{\mathrm{i}}}
$$

The last step includes calculating the value of the statistics for the analysed set of random variable realisations:

$$
\chi_{0}^{2}=\sum_{\mathrm{i}=1}^{\mathrm{n}} \chi_{\mathrm{i}}^{2}=2.8012
$$

The calculated value of the $\chi_{0}^{2}$ statistics should be compared with its critical value determined from the statistical tables [10], at the assumed significance level $\alpha$ and the number of freedom degrees $f$ calculated from the following relation:

$$
\mathrm{f}=1-\mathrm{k}-1
$$

where:

$1-$ number of intervals in the distributive series,

$\mathrm{k}$ - number of estimated parameters of the verified distribution.

Two parameters characterising the normal distribution: $\overline{\mathrm{x}}$ and $\bar{\sigma}$, were evaluated based on the empirical data. For the number of degrees of freedom: $f=2(1=5, k=2)$ and the assumed significance level: $\alpha=0.05$ the critical value of the statistics which was read in the $\chi^{2}$ distribution tables is equal to $\chi_{\mathrm{kr}}^{2}=5.991[10]$.

The calculated value of the statistics is equal to $\chi_{0}^{2}=2.8012$ and is smaller than the critical value $\chi_{\mathrm{kr}}{ }^{2}(\alpha=0.05, \mathrm{f}=2)=5.991$. That means that there is no ground for rejecting the zero hypothesis and, consequently, that the analysed empirical distribution can be considered consistent with the normal distribution. Therefore the probability density function of the random variable $\mathrm{X}(\mathrm{t})=\left\{\mathrm{x}_{\mathrm{i}}\right\}$ in the initial operation stage (time $t=0)$ can be given by the formula:

$$
f(x)=\frac{1}{\sigma \cdot \sqrt{2 \pi}} \cdot \exp \left[-\frac{(x-\bar{x})^{2}}{2 \cdot \sigma^{2}}\right]
$$

Having known the formula for the distribution and its basic parameters we can calculate the limits of the diagnostic tolerance zone assuming the value interval $\mathrm{m} \pm 3 \sigma$ as the diagnostic limiting conditions for the control parameter of interest. For the analysed starting temperature the calculated expected value was equal to $\mathrm{m}=\overline{\mathrm{x}}=18.98 \mathrm{~K} / \mathrm{s}$ and the average deviation was $\sigma=\bar{\sigma}=3.78 \mathrm{~K} / \mathrm{s}$, which gives the following diagnostic tolerances of the analysed control parameter:

$$
7.64 \leq \mathrm{x}_{\mathrm{t}=0} \leq 30.32
$$

It results from the performed calculations that the diagnostic tolerances of the rate of exhaust gas temperature grow in the start-up process of the turbine engine of UGT3000 type are limited from one side, which means that the limiting value of this parameter assessed for diagnostic purposes must not exceed $30 \mathrm{~K} / \mathrm{s}$ in a new engine.

Taking advantage, in turn, of the symmetry of the normal distribution and the values of the Laplace function $\Phi_{p}\left(z_{i}\right)$ we can calculate the probability that the random variable $\mathrm{X}(\mathrm{t})$ takes a value from within this interval:

$$
\mathrm{P}\{\mathrm{X} \in(\mathrm{m} \pm 3 \sigma)\}=\Phi_{\mathrm{p}}(3)-\Phi_{\mathrm{p}}(-3)=0.9973
$$

Thus, assuming the three-sigma limits for the diagnostic tolerances of the analysed control parameter means that less than $3 \%$ ( 3 out of 1000 as the maximum) of possible results of measurement differ from the expected value by more than $3 \sigma$.

In the process of engine operation on a ship, the action of unfavourable operating agents leads to ageing, wear, and pollution of constructional elements. These processes are permanent and irreversible, and they always accompany the operation of internal combustion engines in marine conditions. All this leads to the decrease of the usable potential, the measure of which are the deformations of the shape of probability density function defined by the changes of the expected value and the average deviation, while the normal distribution of the random variable (dispersion of the analysed control parameter) is still being preserved. As a consequence, the dispersion of the exhaust gas temperature grow rate in the engine start-up process increases, and the limits of the diagnostic tolerances, which under no circumstances can exceed the operating tolerances, become wider-Fig. 2. We accept that with the increasing total time of engine operation its functioning is getting less correct, but still preserves all requirements of safe use.

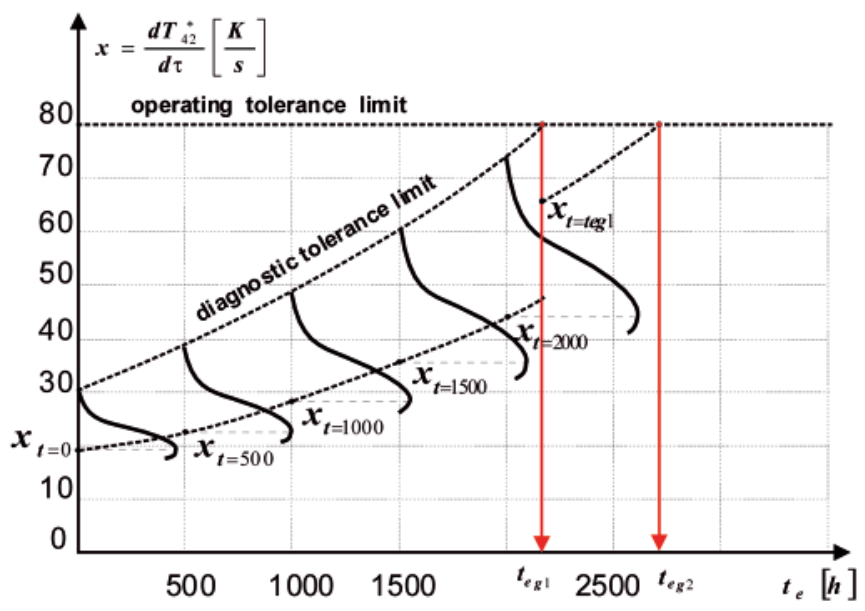

Fig. 2. Deformations of the shape of the probability density function for the analysed random variable vs. the total time of operation of the turbine engine

It results from the numerical data shown in Fig. 1 that after calculating the shapes of the probability density function of the random variable in successive stages of engine operation (for instance, in annual control and measurement cycles, or after each 500 hours of operation) we can determine the trend lines for the changes of the expected value and the upper tolerance limit of the set or realisations of the diagnostic parameter. We can also assess the horizon for the prognosis of correct engine operation (without failures) until time $t_{\text {eg1 }}$ based on the crossing point of the line of upper diagnostic tolerance limit with the line of the operating tolerance limit. For this purpose the value 
of the diagnostic parameter is to be measured on the examined engine - for instance, the point:

$$
\mathrm{x}_{\mathrm{t}=\operatorname{teg} 1}=\left(\frac{\mathrm{dT}_{42}^{*}}{\mathrm{~d} \tau}\right)_{\mathrm{t}=\operatorname{teg} 1}
$$

and the upper diagnostic tolerance limit is to be extrapolated until it crosses the line of the operating tolerance limit (time $t_{\text {eg2 }}$ ) etc. This way we formulate the strategy of engine operation based on its current technical state, the reconstruction of which (adjustment, repair, replacement of an element) depends on individual features of constructional structure degradation, mainly connected with the nature of changes of engine load (number of start-ups, stop-down, accelerations and decelerations of rotor units, manoeuvres of the separate power turbine, etc.).

\section{CONCLUSIONS}

The presented sample application of the statistical method for determining the diagnostic tolerances of the rate of grow of the exhaust gas temperature recorded in the start-up process of a naval turbine engine can be extended onto remaining control parameters recorded during engine operation. A necessary condition for statistical inference is to adopt the assumption that in the initial stage of operation the examined engines maintain correct functioning, which is assessed from the values of basic parameters. In this situation the value of the observed control parameter shifts towards the limit of the diagnostic tolerance zone, thus generating a symptom of small changes of the technical state, characteristic for the "approaching" inability state. Simultaneously it reflects the dependence of the technical state of the engine on the time of its use, which manifests itself by changes of the expected values of the control parameter and its average deviations in successive engine operation stages.

\section{BIBLIOGRAPHY}

1. Balicki W., Szczeciński S.: Diagnosing aircraft turbine engines. Rotor machines. (in Polish). Scientific Library of the Institute of Aviation. Warsaw 2001.

2. Boliński B., Stelmaszczyk Z.: Aircraft propulsion systems. Operation of turbine engines (in Polish). WKiL, Warsaw 1981.
3. Cohen H., Rogers G.F.C., Saravanamuttor H.I.H.: Gas turbine theory. Longman Scientific \& Technical, New York 1987.

4. Dzida M.: Influence of gas turbine controller adjustment on ship propulsion system behavior in rough sea conditions Part 2. The simulation investigations. Polish Maritime Research, No 1(39), 2004, Vol.11.

5. Dzida M.: Simulation of ship propulsion gas turbine dynamics - an educational laboratory model. Polish Maritime Research, No 4(22), 1999, Vol.6.

6. Hardin J.R. and others.: A gas turbine condition - monitoring system. Naval Engineers Journal, November, USA 1995.

7. Korczewski Z.: Method of diagnosing the flow part of a naval turbine engine in operation (in Polish). AMW (Ph.D. thesis), Gdynia 1992.

8. Korczewski Z.: Identifying gasodynamic processes in the compressor system of a naval turbine engine for diagnostic purposes (in Polish). AMW Gdynia 1999.

9. Korczewski Z.: Endoscopy of naval engines (in Polish). AMW Gdynia 2008.

10.Korzyński M.: Methodology of experiment (in Polish). WNT Warszawa 2006.

11. Orkisz M.: Selected problems in the theory of naval jet engines (in Polish). ITE, Radom 1995.

12.Pawlak W., Wiklik K., Morawski J.M.: Synthesis and investigations of aircraft turbine engine control systems using computer simulation methods (in Polish). Institute of Aviation, Warsaw 1996.

13.Szczeciński S.: Aircraft turbine engines (in Polish). Wydawnictwo MON, Warsaw 1965.

14. Technical and operating documentation of naval turbine engines GTU6a, DE59, ZORYA of UGT type, General Electric LM2500 (in Polish).

15.Reports on diagnostic tests of piston and turbine engines in operation on Polish Navy vessels - Research activities of AMW (in Polish), Gdynia $1992 \div 2008$.

\author{
CONTACT WITH THE AUTHOR \\ Zbigniew Korczewski, Prof. \\ Faculty of Ocean Engineering \\ and Ship Technology \\ Gdansk University of Technology \\ Narutowicza 11/12 \\ 80-233 Gdansk, POLAND \\ e-mail: z.korczewski@gmail.com
}

